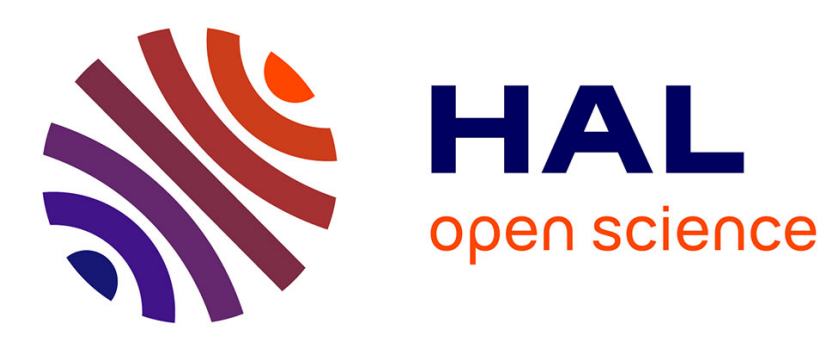

\title{
A quantitative morphological analysis of nanostructured ceria-silica composite catalysts
}

\author{
M. Moreaud, Dominique Jeulin, Alain Thorel, J.-Y. Chane-Ching
}

\section{To cite this version:}

M. Moreaud, Dominique Jeulin, Alain Thorel, J.-Y. Chane-Ching. A quantitative morphological analysis of nanostructured ceria-silica composite catalysts. Journal of Microscopy, 2008, 232 (2), pp.293-305. 10.1111/j.1365-2818.2008.02101.x . hal-00359123

\section{HAL Id: hal-00359123 \\ https://hal.science/hal-00359123}

Submitted on $10 \mathrm{Jul} 2018$

HAL is a multi-disciplinary open access archive for the deposit and dissemination of scientific research documents, whether they are published or not. The documents may come from teaching and research institutions in France or abroad, or from public or private research centers.
L'archive ouverte pluridisciplinaire $\mathbf{H A L}$, est destinée au dépôt et à la diffusion de documents scientifiques de niveau recherche, publiés ou non, émanant des établissements d'enseignement et de recherche français ou étrangers, des laboratoires publics ou privés. 


\title{
A quantitative morphological analysis of nanostructured ceria-silica composite catalysts
}

\author{
M. MOREAUD*, D. JEULIN*, A. THOREL $\dagger$ \\ \& J. Y. CHANE-CHING $\ddagger$ \\ ${ }^{*}$ Center of Mathematical Morphology, Mathematics and Systems, MINES ParisTech, 35 rue Saint \\ Honoré, 77300 Fontainebleau, France \\ $\dagger$ Center of Materials Sciences, MINES, ParisTech, U.M.R. CNRS 7633, B.P. 87, 91003 Evry, \\ France \\ †CIRIMAT/LCMIE - UMR CNRS 5085, University Paul Sabatier, 118, route de Narbonne, \\ 31062 - Toulouse CEDEX 9, France
}

Key words. Catalyst, ceria, HRTEM, interclass variance maximization, mathematical morphology, mesoporous material.

\begin{abstract}
Summary
This study aims at examining the morphology of different catalysts, which are based on a dispersion of ceria nanoparticles embedded in a high surface area mesoporous silica framework. In order to fully describe the mesostructured composite material, we propose here a quantitative description of the microstructure based on a quantitative analysis of micrographs that were obtained via high-resolution transmission electron microscopy. We have therefore developed an automatic image analysis process in order to automatically and efficiently extract all the components of the catalyst images. A statistical and a morphological analysis of the spatial arrangement of the components of the catalyst are also presented. The study shows clear differences between the materials analysed in terms of the spatial arrangement and the total surface area of the ceria phase emerging into the pores, parameters of prime importance for the catalytic properties. Thus, the silica-ceria nanostructured composite materials, displaying large surface area up to $300 \mathrm{~m}^{2} \mathrm{~g}^{-1}$ are shown to exhibit highly rugged surfaces resulting from ceria nanoparticles emerging in the pores.
\end{abstract}

\section{Introduction}

Mesoporous materials are the subject of many studies and triggered a strong interest in the field of catalysis (Nooney et al., 2002; Che et al., 2003; Tian et al., 2003; Corma et al., 2004a). Composite mesostructured silica-ceria catalysts have been proposed (Chane-Ching, 1999; Corma et al., 2004b), in which

Correspondence to: Dominique Jeulin. Tel: (33) 1646947 95; fax: (33) 164 6947 07; e-mail: dominique.jeulin@ensmp.fr the catalytic functionality is ensured by the ceria nanoparticles emerging into the pores that originate from the calcination of the copolymer template arranged in a hexagonal structure. To improve the catalytic functionality of the material, one needs to characterize precisely the dispersion and volume fraction of both the ceria particles and the pore channels within the material, to geometrically cross-correlate and relate them to the manufacture process. Various mesoporous catalysts corresponding to various manufacture process conditions were recently proposed (Chane Ching, 1999). To characterize their morphology, the materials were observed by means of transmission electron microscopy (TEM). TEM micrographs were analysed with techniques based on statistical and mathematical morphology tools (Moreaud, 2006) in order to efficiently and automatically extract the various components of the catalysts images (see Fig. 1): the pores, silica and the ceria nanoparticles. In this paper, we will present the results of such an analysis obtained for three kinds of catalysts (mat. 1,2 and 3) with three sets of five high-resolution TEM images (sets 1,2 and 3).

\section{Presentation of the catalysts}

The fabrication of the silica-ceria catalysts involves the self-assembly of $\mathrm{CeO}_{2}$ monodisperse nanoparticles and a $\mathrm{SiO}_{2}$ binder from an acidic homogenous medium (cf. Fig. 1). The assembly process was controlled by adjusting the interactions between a copolymer template with the $\mathrm{CeO}_{2}$ nanoparticles and $\mathrm{SiO}_{2}$ (Corma et al., 2004a, 2004b). The catalytic functionality of these materials is ensured by the insertion of 5-nm-wide ceria nanoparticles in the copolymer network during the mineralization process. The copolymer network is then calcined, giving rise to a hexagonal network 
(a) Hexagonal network of co-polymer pencils

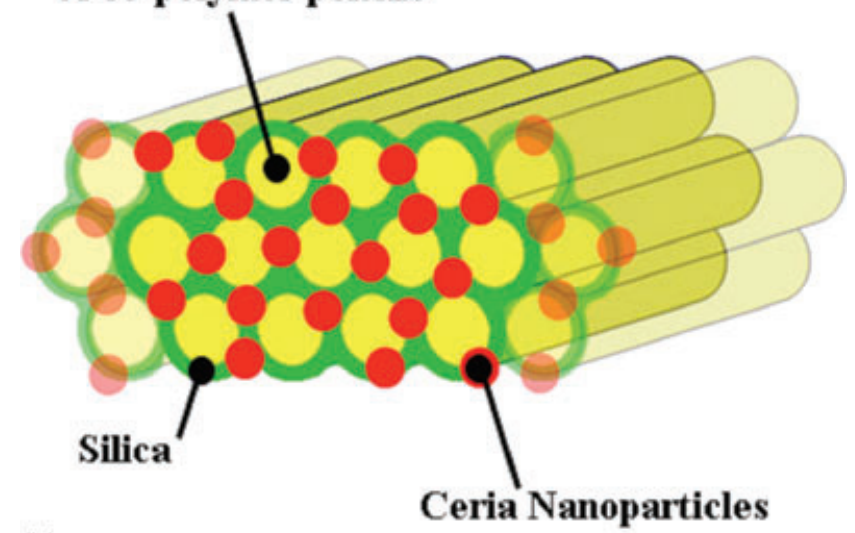

(b)

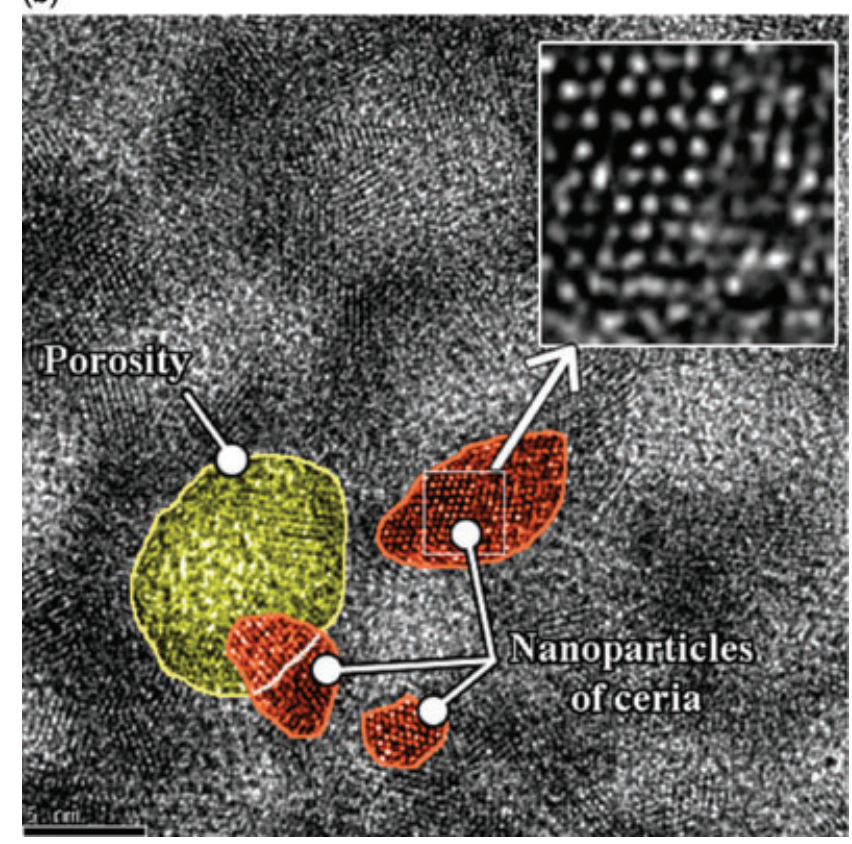

Fig. 1. Left, representation of the composition of the catalysts. Right, image obtained by transmission electron microscopy (in top corner right, we can observe the atomic structure of a ceria nanoparticle). (Image $=$ $512 \times 512$ pixels, numerical resolution $=0.07 \mathrm{~nm}$.)

of porous channels, in which a certain volume fraction of ceria emerges (Shinkai et al., 2003; Chane-Ching et al., 2005). The manufacture process and hence the properties of these catalysts can be significantly improved from morphological information on the ceria nanoparticles and their distribution (Nooney et al., 2002): compactness, sphericity and localization either in the pores and or in the silica walls.

\section{Preparation of TEM samples and observations}

Three materials corresponding to three different volume fraction of ceria were impregnated with a resin and cut in slices with a thickness of a few tens of nanometres by ultra-microtome. In order to avoid any skew, the exact volume fraction of ceria for each materials was not known by those who carried out the numerical analysis. Samples were observed with a TEM (Tecnai 20F ST, FEI Europe, Eindhoven, The Netherlands) operated at $200 \mathrm{kV}$ under conditions close to the Scherzer defocus. These observations were carried out in the Centre des Matériaux de l'Ecole des Mines de Paris (Thorel \& Molins, 2003). The spatial resolution of the 256 grey levels images is $0.07 \mathrm{~nm}$ per pixel. The actual resolution of the micrographs (five per material) corresponds to the resolution of the microscope, and is of the order of $0.2 \mathrm{~nm}$, making possible a precise localization of the ceria nanoparticles.

\section{Image processing}

We present below a set of image processing tools, which have been developed to carry out the segmentation of pores, resin, silica and ceria nanoparticles in three sets of high-resolution TEM images (sets 1, 2 and 3) corresponding to three materials (mat. 1, 2 and 3).

\section{Segmentation of the porosity network}

The pores are filled with resin with very little electron scattering capability, and therefore appear as light grey aggregates in HRTEM images. To extract them, we use mathematical morphology tools and an automatic binary segmentation. We process a morphological opening by a disk of diameter $0.7 \mathrm{~nm}$, and then we use binary thresholding by maximization of the interclass variance (Otsu, 1979). With this first step, we obtain an image $I_{\mathrm{m}}$, which is a marker that will be used to extract the pores aggregates.

$$
I_{\mathrm{m}}=S\left(\left(I-D_{0.7}\right) \oplus D_{0.7}, s_{0}\right)
$$

with $I$ the initial image, $D_{0.7}$ a disk of diameter $0.7 \mathrm{~nm}$, $S$ the result of a binary thresholding operator and $s_{0}$ the threshold obtained by maximization of the interclass variance. The binary image $I_{\mathrm{p}}$, which gives a precise representation of pores, is obtained in two steps:

(1) First, we generate a morphological reconstruction (Serra, $1982)$ of image $S\left(I, s_{0}\right)$ from the marker $I_{\mathrm{m}}, S\left(I, s_{0}\right)$ being the initial image after binary thresholding by the interclass variance maximization.

(2) Then, we perform a morphological closing by a disk of diameter $0.37 \mathrm{~nm}$. This step is used to eliminate holes that could remain in the resin (pores).

$$
I_{\mathrm{p}}=\left(\operatorname{Rec}_{\oplus}\left(I_{\mathrm{m}}, S\left(I, s_{0}\right)\right) \oplus D_{0.35}\right)-D_{0.35}
$$

$\operatorname{Rec}_{\oplus}$ is the morphological reconstruction operator. The process is illustrated in Fig. 2. 
(a)

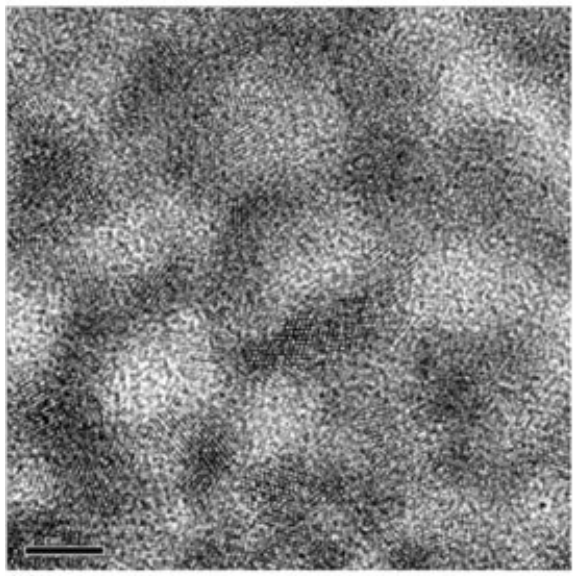

(c)

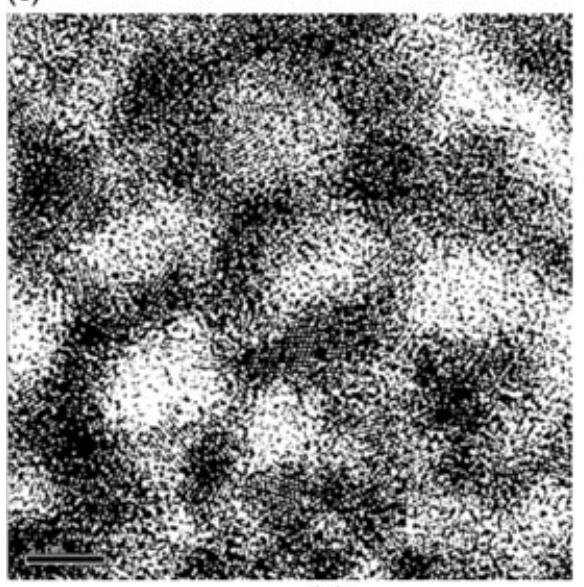

(b)

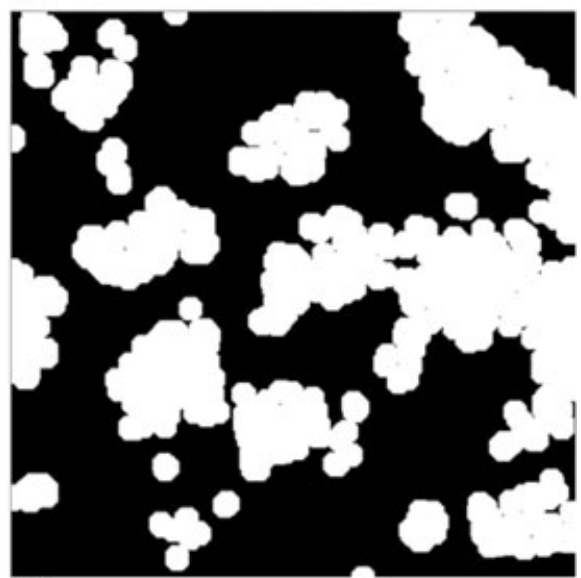

(d)
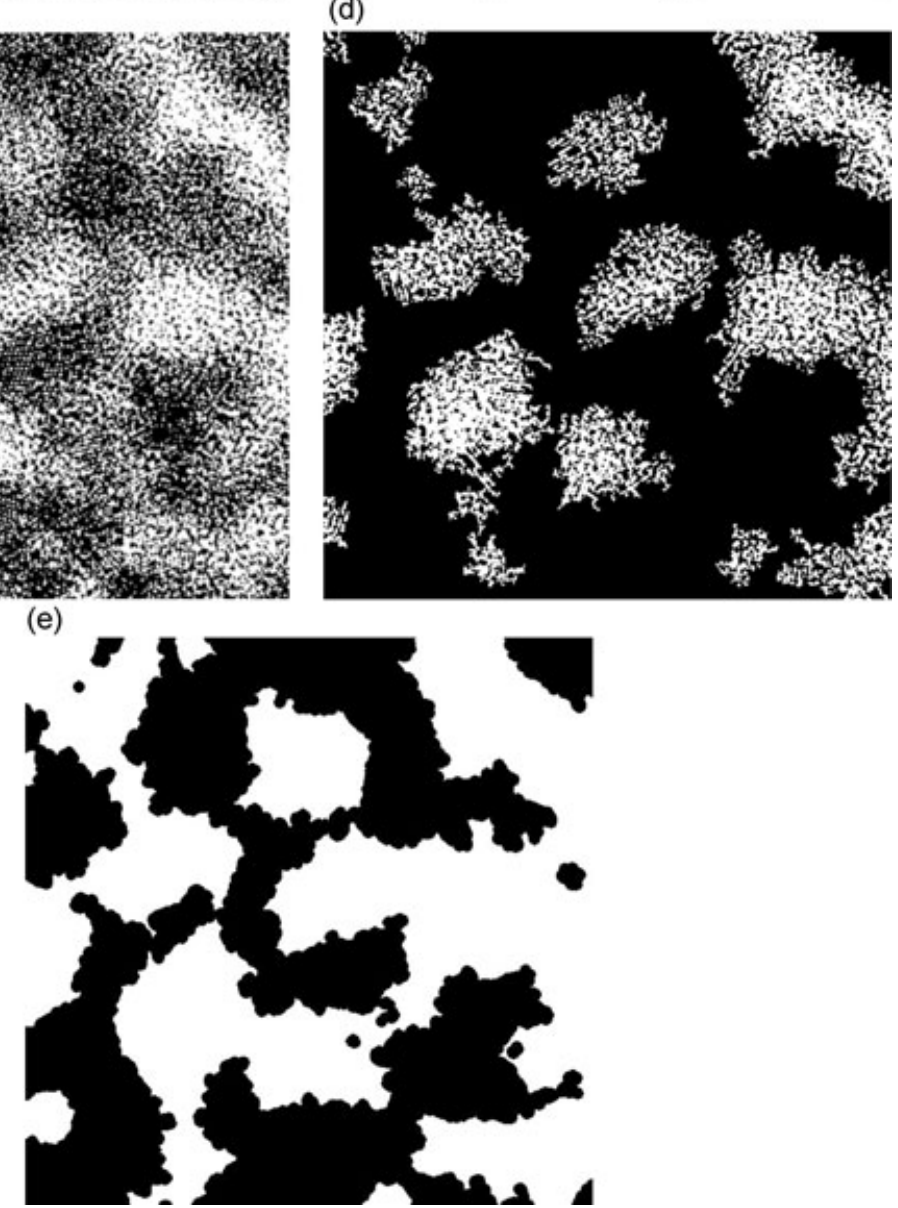

Fig. 2. The various steps to obtain the binary image of pores $I_{\mathrm{p}}$ (example on an image of set 1 ). From left to right, and from top to bottom: initial image $I$, image $I_{\mathrm{m}}$, image $S\left(I, s_{0}\right)$, image $\operatorname{Rec}\left(I_{\mathrm{m}}, S\left(I, s_{0}\right)\right)$, and image $I_{\mathrm{p}}$ corresponding to the image of pores. (The images consist of $512 \times 512$ pixels, numerical resolution $=0.07 \mathrm{~nm}$.)

\section{Detection of silica and ceria nanoparticles}

The ceria nanoparticles and the silica walls correspond to areas in the images that present little white spots with sizes ranging from 0.2 to $0.5 \mathrm{~nm}$. These spots are associated with the periodic arrangement typical of the phase contrast structure image for crystalline ceria, and with the short-range arrangement of randomly distributed interconnected $\mathrm{SiO}_{4}$ tetrahedrons for vitreous silica observed under phase contrast conditions. To extract these spots, we make a convolution product of the 
(a)

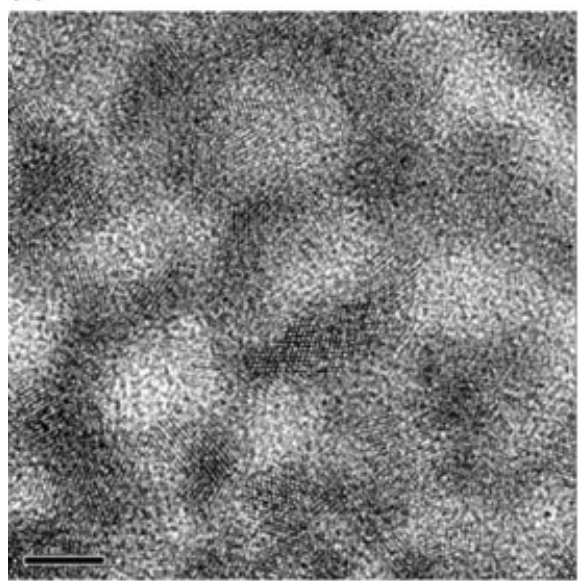

(c)

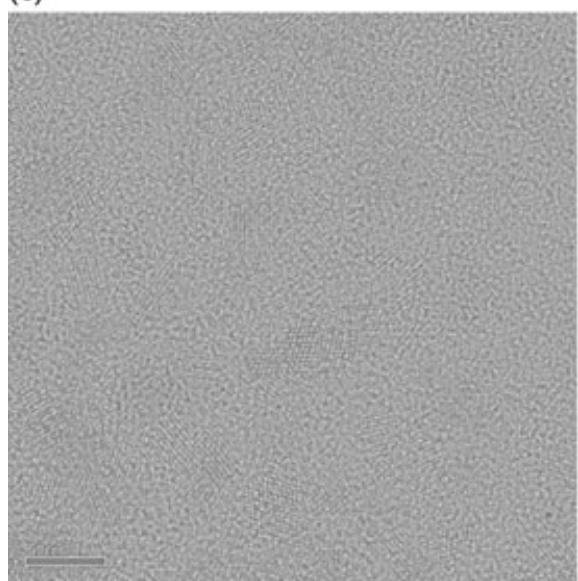

(b)

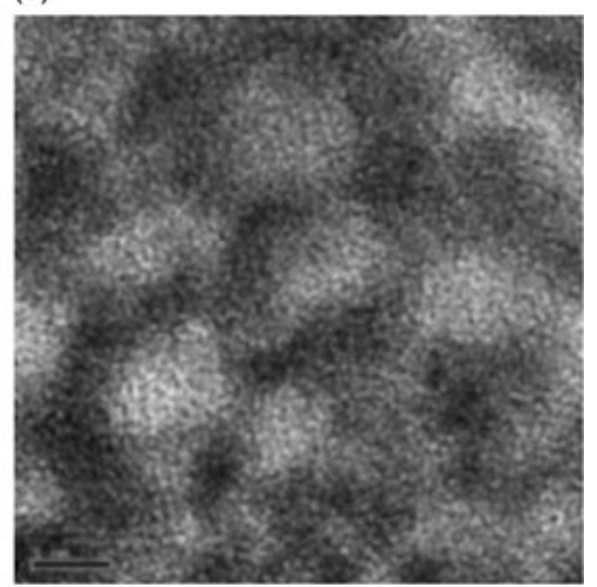

(d)

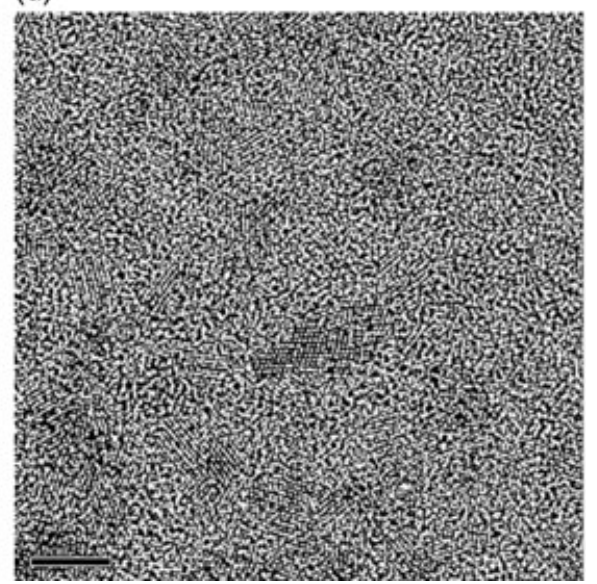

Fig. 3. Extraction of the silica and ceria phases (example on an image extracted from set 1). From left to right, and from top to bottom: initial image $I$, image $I_{\mathrm{g}}$ (smoothing by a Gaussian filter), $I-I_{\mathrm{g}}$ (image of the "details" of $I$ ), final image $I_{\mathrm{sc}}$ (binary thresholding of $I-I_{\mathrm{g}}$ by maximization of the interclass variance). (The images consist of $512 \times 512$ pixels, numerical resolution $=0.07 \mathrm{~nm}$.)

initial image $I$ by a Gaussian normalized and centred kernel $G(x, y)$ with size $0.5 \times 0.5 \mathrm{~nm}$ and with a standard deviation equals to $3.5 \mathrm{~nm}$. The image $I_{\mathrm{g}}$, is obtained, which corresponds to the initial image from which all the details of less than $0.5 \mathrm{~nm}$ wide are removed.

$$
\begin{gathered}
G(x, y)=\frac{1}{2 \pi \sigma^{2}} \mathrm{e}^{-\frac{x^{2}+y^{2}}{2 \sigma^{2}}}=\frac{1}{76.97} \mathrm{e}^{-\frac{x^{2}+y^{2}}{24.5}} \\
\text { for } x, y \in[-0.5 \mathrm{~nm}, 0.5 \mathrm{~nm}] \\
I_{\mathrm{g}}=I * G
\end{gathered}
$$

We then carry out a subtraction between $I$ and $I_{\mathrm{g}}$ to obtain the details that are missing in I. After thresholding this result by maximization of the interclass variance, we obtain the binary image $I_{\mathrm{sc}}$ of the silica and ceria phases.

$$
I_{\mathrm{sc}}=S\left(I-I_{\mathrm{g}}, s_{0}\right)
$$

An illustration of this process can be seen in Fig. 3.

\section{Separation of silica and ceria phases}

At the end of the previous step, we obtain a binary image containing pixels that correspond either to the silica phase or to the ceria phase. To separate these two phases, we use the fact that ceria is crystallized with a large inter-reticular parameter that allows the material to diffract strongly; furthermore, owing to the very small size of the ceria nanoparticles (typically $5 \mathrm{~nm}$ ), the Bragg's conditions are somehow relaxed, and whatever their orientation, the ceria nanoparticles always present a zone axis close to the MET optical axis, or at least a set of reticular planes parallel to the optical axis, which always leads to the presence of a visible periodicity when the ceria nanoparticles are observed under HRTEM conditions. We have checked by contrast simulation (software CrystalKit and MacTempas) that any tilting of a ceria nanoparticle by an angle at most equal to half the angle between two zone axes in the structure led to a periodic image for the ceria particles that could be easily segmented via a Fourier analysis. 
(a)

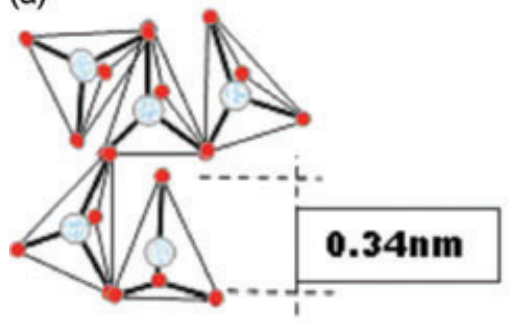

(b)
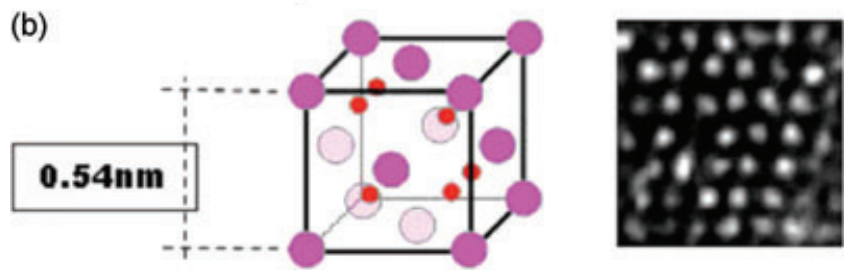

Fig. 4. Difference in the atomic organization between silica and the ceria nanoparticles. Left: silica. We can notice short distance order and reticulation by the summits of the $\mathrm{SiO}_{4}$ tetrahedrons. Right: ceria nanoparticles. We can observe periodicity of the unit cell and HRTEM image of the atomic structure.

Silica is vitreous, without periodicity, but its structure exhibits a short distance order because of the $\mathrm{SiO}_{4}$ silica tetrahedron geometry, and a coherence length associated with the fact that these $\mathrm{SiO}_{4}$ tetrahedrons are randomly connected and reticulated by their summits in the silica vitreous structure (cf. Fig. 4). It is beyond the scope of this paper to make the full interpretation of the atomic structure images of either ceria or silica phases; it is only important to consider here that the HRTEM images of the ceria phase present a systematic periodic contrast, that the HRTEM images of the silica phase present a characteristic coherence length with no periodic contrast, and that our HRTEM observations have been recorded at a defocus close to the Scherzer defocus. A local Fourier transform highlights these phenomena; for ceria nanoparticles, several peaks are systematically observed in the Fourier space in addition to the central peak, whereas a strong central peak is observed in the Fourier space for silica, along with a very weak centred halo that corresponds to a spatial frequency associated with the silica real space coherence length (cf. Fig. 5).

We propose the following method to extract ceria: we take for each pixel $(X, Y)$ a centred neighbourhood on which we perform a fast Fourier transform (FFT). Then, we apply a binary thresholding to the power spectrum image calculated by FFT with a threshold equal to a fraction of the value of the intensity of the central peak. If additional peaks are found in this binarized spectrum image, then the pixel $(X, Y)$ belongs to ceria and is given a white contrast; if no additional peak is found in the Fourier space, then the pixel $(X, Y)$ belongs to silica and is attributed a black contrast. At the end of this process, we obtain the image $I_{\mathrm{c}}$ in which all the white pixels represent the ceria nanoparticles. After several trials for fine-tuning the parameters of the process (size and shape of the window for the FFT, and fraction of the value of the central peak), we have determined what were the optimal parameters from a visual comparison between the original and the segmented images (cf. Fig. 6). This process is subjective, but very common to evaluate the quality of image segmentation. The size of the window for the FFT is $2.24 \times 2.24 \mathrm{~nm}$ and the threshold is equal to the value of the intensity of the central peak divided by 4.5 . These parameters have been kept the same for all the processed images. The spatial arrangement of silica can be easily obtained from the complementary image of $I_{\mathrm{c}}: I_{\mathrm{s}}: I_{\mathrm{c}}{ }^{\mathrm{c}}$.

\section{Results of the processing of images}

We have shown that an efficient segmentation of the pores, the silica and the ceria nanoparticles can be achieved. As a first outcome, an image showing the overlapping of the different segmented phases can be reconstructed, from which it is seen that a qualitative information regarding the relative location of ceria and pores can be straightforwardly drawn (Fig. 7). More interestingly, we will demonstrate in the next sections of the present paper that the phases' segmentation enables us to make all kinds of measurements, and in particular a relevant evaluation of the volume fraction of ceria emerging in the pores - results of prime importance as far as the fabrication process optimization is concerned.

\section{Analysis of segmented images}

After processing the TEM micrographs, we have obtained the various components of the catalysts: the pores originating from the co-polymers network on the image $I_{\mathrm{p}}$, the ceria nanoparticles on the image $I_{\mathrm{c}}$ and the silica walls from the complementary image of $I_{\mathrm{c}}: I_{\mathrm{S}}: I_{\mathrm{c}} \mathrm{c}$.

\section{Isotropy and homogeneity of the spatial distribution of ceria nanoparticles}

From the estimated covariance function which was averaged over horizontal and vertical directions of measurement, we get information on the anisotropy of the microstructure, because the covariance of an isotropic material is invariant by rotation (Matheron, 1975; Jeulin, 1991; Serra, 1982). The covariance $C(h)$ is defined as the probability for a pair of points $\{x, x+h\}$ to belong to a set $\mathrm{A}$ (for instance one component of the material). It depends on the vector $h$ :

$$
C(h)=P\left\{x \in A \cap A_{-h}\right\}
$$

with $A_{-h}$ the set $A$ translated of the vector $-h$

We have measured the covariance in two directions (horizontal and vertical) for the five images of each set, and we have calculated the average function. The plots of the covariance in the two directions are compared for each of the 
(a)

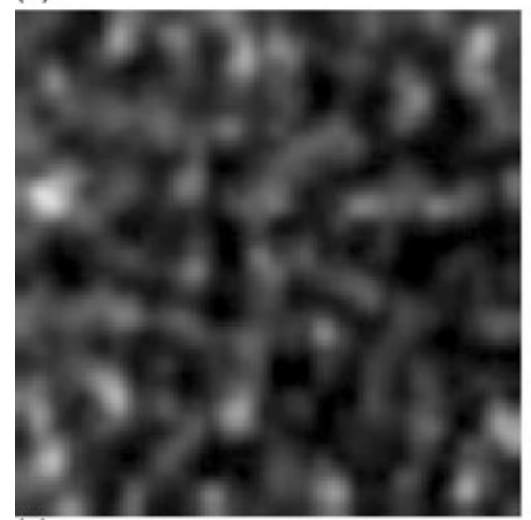

(c)

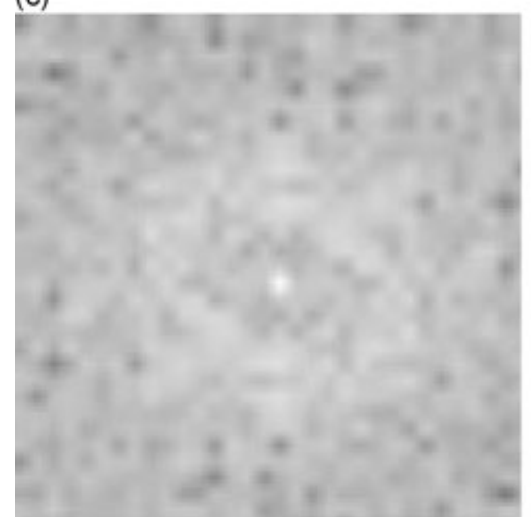

(e)

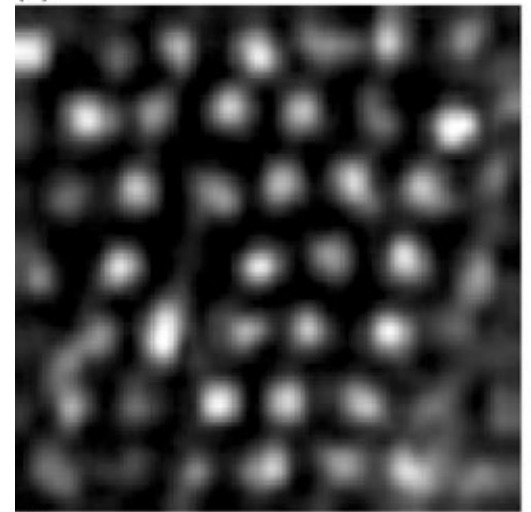

(g)

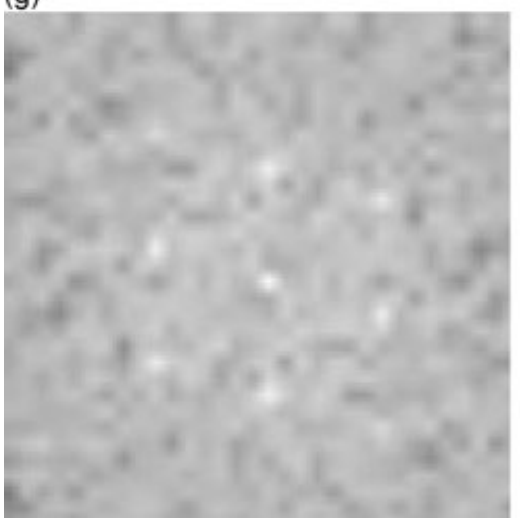

(b)

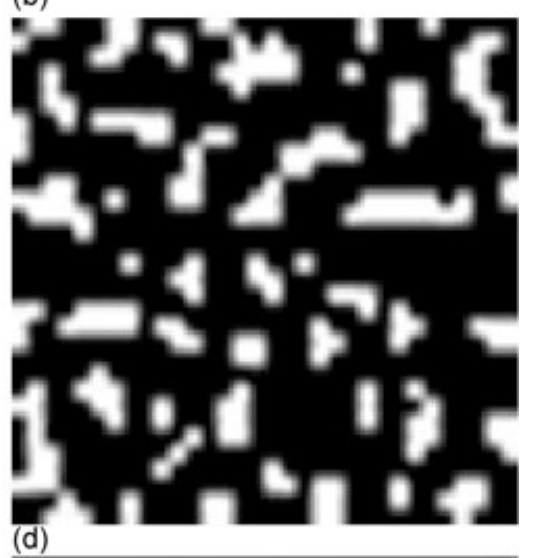

(d)
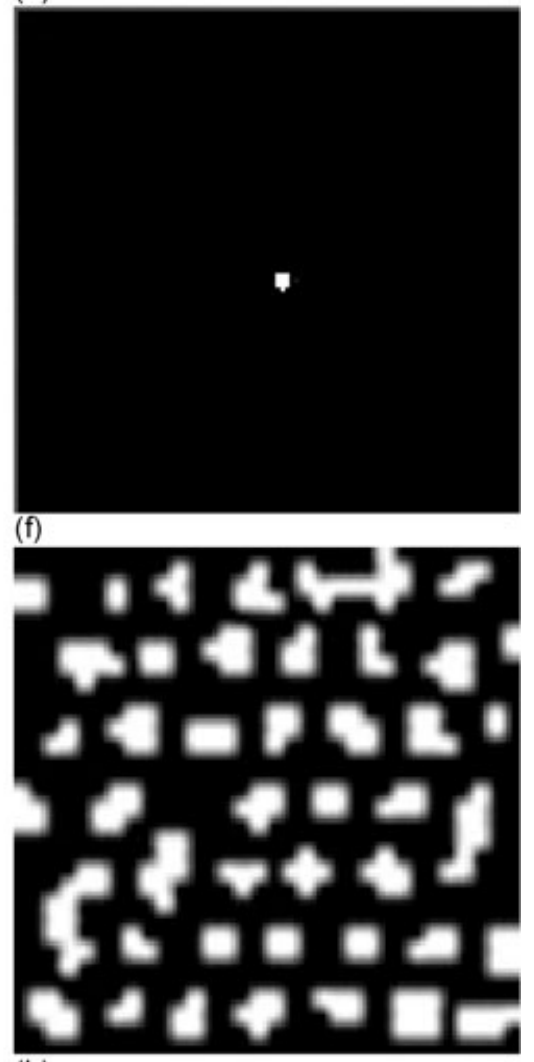

(h)

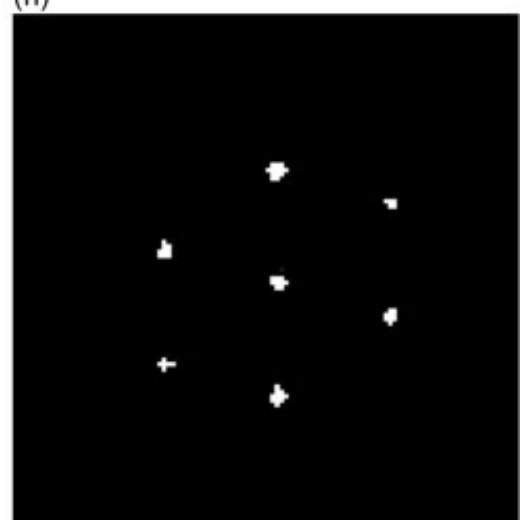

Fig. 5. Comparison between silica (top) and ceria (bottom). From left to right: initial image $I$, image with silica and ceria phases $I_{\mathrm{sc}}$, image of the power of fast Fourier transform (FFT) of $I_{\mathrm{sc}}$, thresholding of the power of the FFT. Size of the initial images: $2.24 \times 2.24 \mathrm{~nm}^{2}$. 
(a)

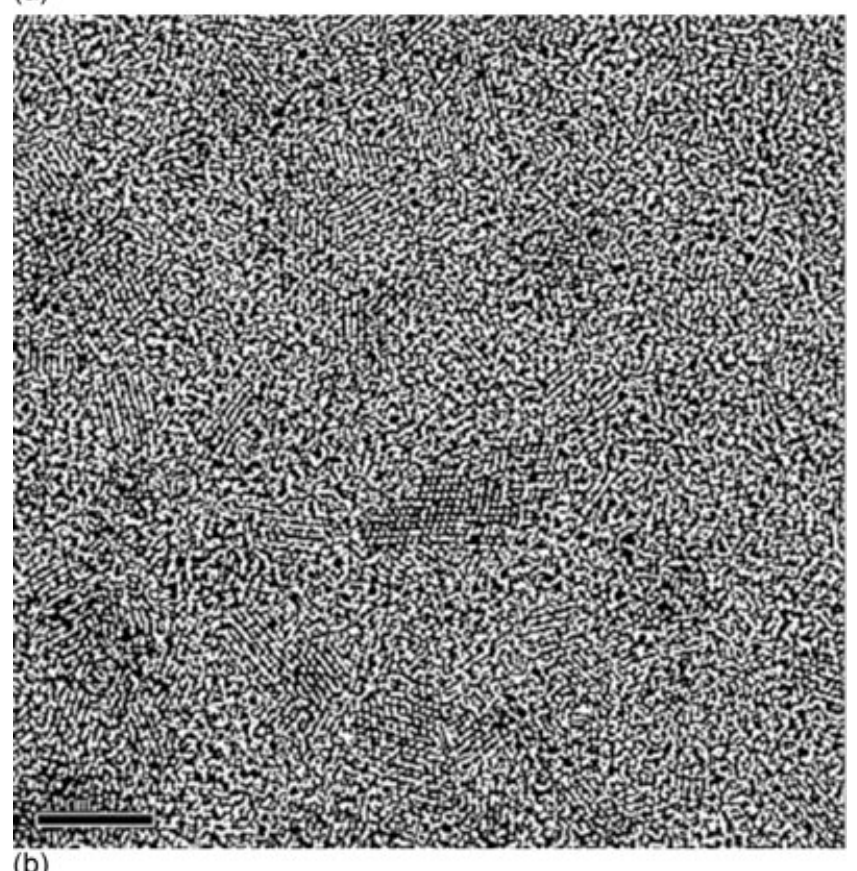

(b)

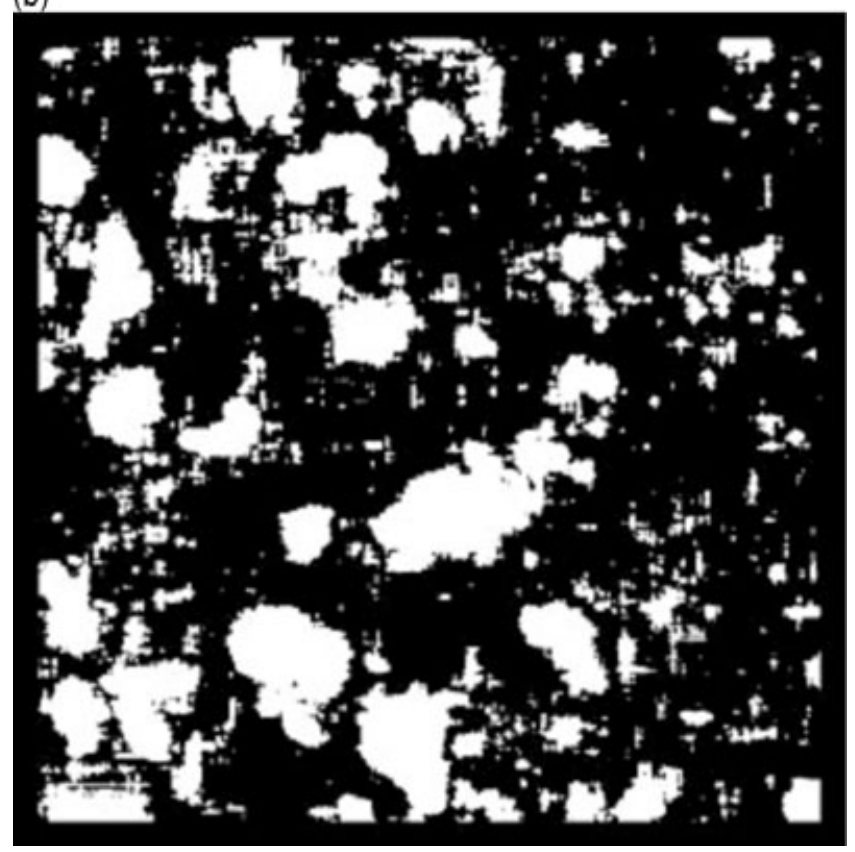

Fig. 6. Separation of the silica and ceria phases. Example of an image extracted from set 1 . At left, image $I_{\mathrm{sc}}$ indicating the silica and ceria phases. At right, image $I_{\mathrm{c}}$, extraction of the ceria phase only (the images consist of $512 \times 512$ pixels, numerical resolution $=0.07 \mathrm{~nm})$.

three materials under considerations: they are quite similar, up to extremely small statistical fluctuations (Fig. 8), indicating that the spatial distribution of the ceria nanoparticles is isotropic for the three catalysts.

In addition, because no periodicity or oscillation appears on the experimental covariance plots, it can be inferred that the ceria nanoparticles are randomly distributed (cf. Fig. 8).

The volume fractions of ceria nanoparticles of catalysts of sets 1, 2 and 3 are $33 \%, 61 \%$ and $59 \%$, respectively (estimated directly by the value of $C(0)$ ). The catalysts of sets 2 and 3 are appreciably richer in ceria.

\section{Mean size of nanoparticles of ceria}

The mean size of ceria domains can be obtained by measuring the range of the three experimental covariance curves described above. It corresponds to the mean characteristic length of the analysed structure. The range is the value of $h$ when the covariance reaches its asymptotic level given by $C(0)^{2}$. We obtain for ceria a range close to $4.4 \mathrm{~nm}$ for set 1 , $5.2 \mathrm{~nm}$ for set 2 and $5.3 \mathrm{~nm}$ for set 3 (see Fig. 8). These results are consistent with the 5-nm-diameter ceria nanoparticles that were inserted in the starting materials at the mineralization step of the fabrication process.

Additional information is given by the size distribution function of ceria nanoparticles $G(t)$, estimated from the volume fraction $\mathrm{Vv}$ after openings by disks with increasing diameters (Serra, 1982):

$$
G(t)=\operatorname{Vv}\left(I_{\mathrm{c}}\right)-\mathrm{Vv}\left(I_{\mathrm{c}}-D_{t} \oplus D_{t}\right)
$$

$G(t)$ is measured for the five images $I_{c}$ of each set, for which we calculate the mean value. For the three sets, we observe a linear size distribution function at the beginning of the curves of the functions (see Fig. 9), corresponding to a quasi-uniform distribution of sizes of the ceria domains.

\section{Analysis of the pores}

Using the images $I_{\mathrm{p}}$, the volume fraction $\mathrm{Vv}$ of the pores can be estimated. The three catalysts contain approximately $33 \%$ of pores for set $1,40 \%$ for set 2 and $46 \%$ for set 3 .

We have checked that pores formed a quasi-periodic network (periodicities are visible on the covariance curves). The periodicity of the network is approximately 11 and $10 \mathrm{~nm}$ for the catalysts of sets 1 and 2, respectively. For set 3, the pores network behaves differently: a quasi-periodicity is observed in only one direction, with a period of $11 \mathrm{~nm}$ (see Fig. 10). For the catalyst of sets 1 and 3 the range of the covariance of pores is approximately $4.5 \mathrm{~nm}$, versus $3.5 \mathrm{~nm}$ for the catalyst of set 2 (see Fig. 10). This quasi-periodicity is the result of the initial distribution of the co-polymer pencils in the material, as illustrated in Fig. 1.

\section{Distribution of the ceria nanoparticles compared to the pores}

One of the important outcomes of the present study is the estimation of the relative location of the ceria nanoparticles and of the porous network. With the images $I_{\mathrm{p}}$ (corresponding to the pores $P$ ) and $I_{\mathrm{c}}$ (corresponding to the ceria nanoparticles C), and by averaging the results obtained for the five images 


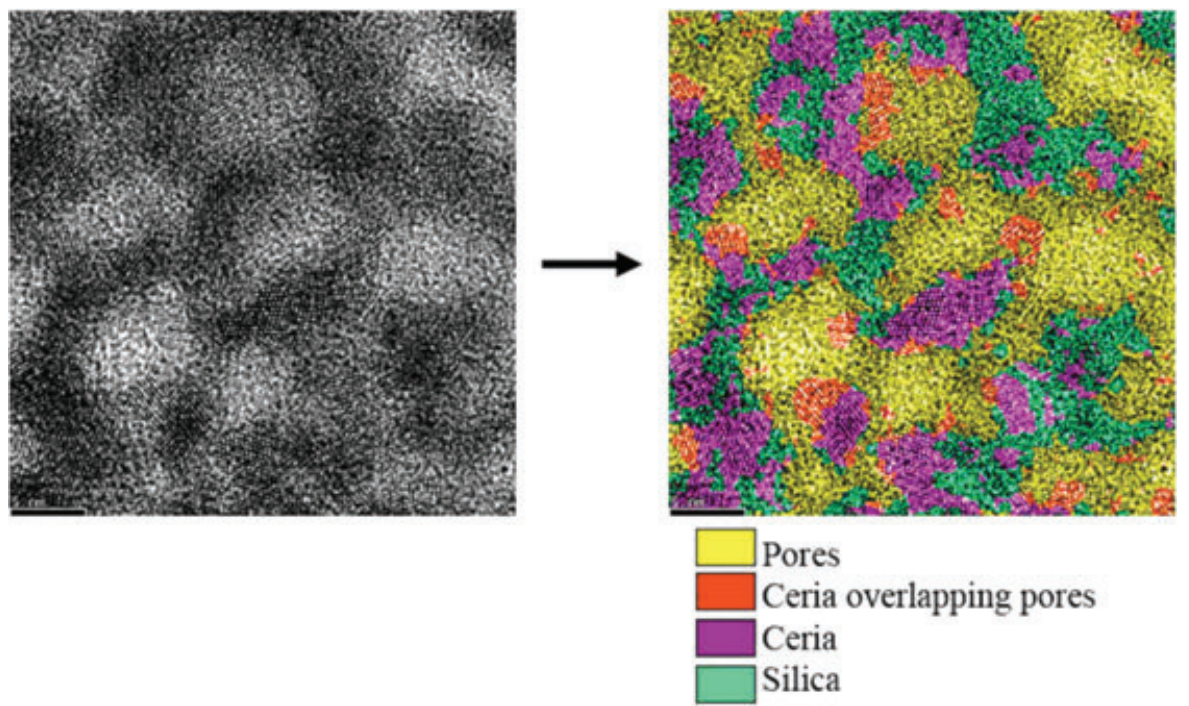

Fig. 7. Segmentation of an image extracted from set 1 (the images consist of $512 \times 512$ pixels, numerical resolution $=0.07 \mathrm{~nm}$ ).

of each set, the volume fractions of ceria nanoparticles inside and outside the pores can be estimated (see Table 1). First, we notice that for the three catalysts the volume fraction of ceria overlapping the pores is significant: $6 \%, 20 \%$ and $22 \%$ for the catalyst of sets 1,2 and 3, respectively. Secondly, $33 \%$ and $38 \%$ of the volume fraction of the ceria particles in catalysts of sets 2 and 3 overlap the pores, against only $18 \%$ for the catalyst of set 1 . In addition, $49 \%$ and $48 \%$ of the volume of the pores contain ceria for the catalysts of set 2 and 3 against $17 \%$ for the catalyst of set 1 . Thus it can be inferred that an increasing amount of ceria allows for an increasing amount of ceria nanoparticles overlapping the pores.

With the images $I_{\mathrm{p}}$ and $I_{\mathfrak{c}}$, we can also estimate the following distributions (Jeulin, 1986):

(1) $F(P, r)$, distribution of distances of a point taken randomly outside the pores to the boundary of the pores

$F(P, r)=P\left\{d(x, P)<r \mid x \in P^{\mathrm{c}}\right\}=\frac{V_{\mathrm{V}}\left(I_{\mathrm{P}} \oplus D_{\mathrm{r}}\right)-V_{\mathrm{V}}\left(I_{\mathrm{P}}\right)}{1-V_{\mathrm{V}}\left(I_{\mathrm{P}}\right)}$

(2) $F_{\mathrm{C}}(P, r)$, distribution of distances of a point taken randomly inside the ceria nanoparticles to the boundary of the pores

$$
F_{\mathrm{C}}(P, r)=P\{d(x, P)<r \mid x \in C\}=\frac{V_{\mathrm{V}}\left(\left(I_{\mathrm{P}} \oplus D_{\mathrm{r}}\right) \cap I_{\mathrm{C}}\right)}{V_{\mathrm{V}}\left(I_{\mathrm{C}}\right)}
$$

(3) $F_{\mathrm{C}}\left(P^{\mathrm{c}}, r\right)$, distribution of distances of a point taken randomly inside the ceria nanoparticles to the boundary of the complementary of the pores

$F_{\mathrm{C}}\left(P^{\mathrm{c}}, r\right)=P\left\{d\left(x, P^{\mathrm{c}}\right)<r \mid x \in C\right\}=\frac{V_{\mathrm{V}}\left(\left(I_{\mathrm{P}}^{\mathrm{C}} \oplus D_{\mathrm{r}}\right) \cap I_{\mathrm{C}}\right)}{V_{\mathrm{V}}\left(I_{\mathrm{C}}\right)}$
We can define a new criterion based on attraction or repulsion between ceria and pores:

$$
\rho_{\mathrm{C}}(P, r)=\frac{F_{\mathrm{C}}(P, r)}{F(P, r)}
$$

If $\rho_{\mathrm{C}}(P, r)$ is larger than 1 , a strong attraction at the distance $r$ between the pores and the ceria nanoparticles is observed. If $\rho_{\mathrm{C}}(P, r)$ is less than 1 , then a repulsion effect at the distance $r$ exists between the ceria nanoparticles and the pores.

If we analyse the curves $F_{\mathrm{C}}\left(P^{\mathrm{c}}, r\right)$ and $F_{\mathrm{C}}(P, r)$ obtained for the three sets of catalysts, one can notice that the ceria nanoparticles of the catalyst of sets 2 and 3 are closer to the pores than the ceria nanoparticles of catalyst of set 1. It appears that $50 \%$ of the fraction of the ceria nanoparticles is inside the pores or at a distance to the boundary of the pores less than $0.5 \mathrm{~nm}$ for the catalysts of sets 2 and 3 , against $1.1 \mathrm{~nm}$ for catalyst of set 1 (see. Fig. 11). Concerning the curves $\rho_{\mathrm{C}}(P, r)$, a strong attraction between the ceria nanoparticles and the pores is also seen for the three sets of catalysts (Fig. 12). Moreover, the catalysts of sets 2 and 3 present a stronger ceria-pores attraction than the catalyst of set 1 . This observation reinforces the fact that an increasing amount of ceria nanoparticles gives rise to an increasing amount of ceria nanoparticles overlapping the pores.

\section{Summary of the results}

With the analysis developed in this paper, we have attained the following results listed in Table 2 . The quasi-periodic pores network characterized by our analysis is in agreement with the suggestion of a structure with well-defined hexagonal symmetry obtained by small-angle X-ray scattering (ChaneChing et al., 2005). We estimate the pores diameter within a 
(a)

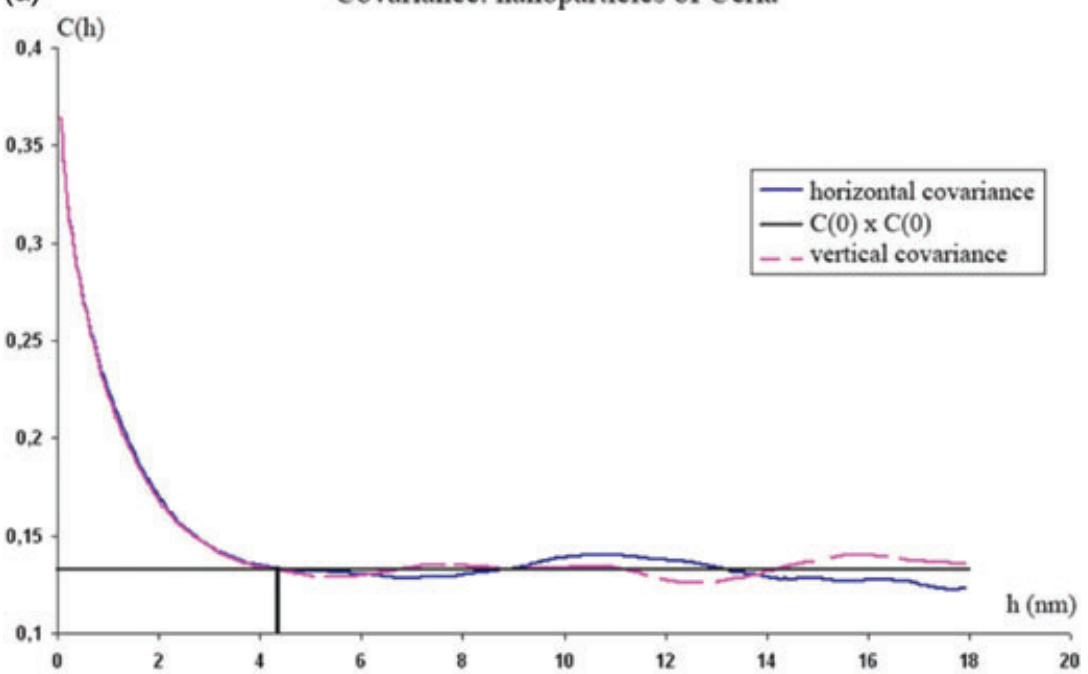

(b)

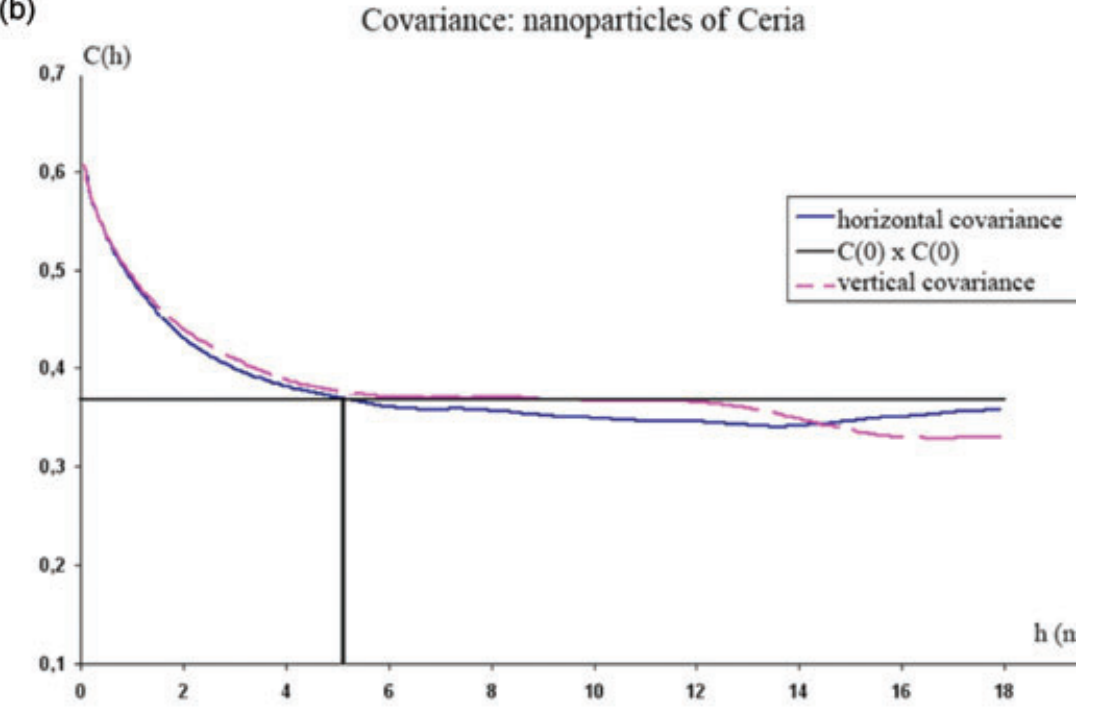

(c)

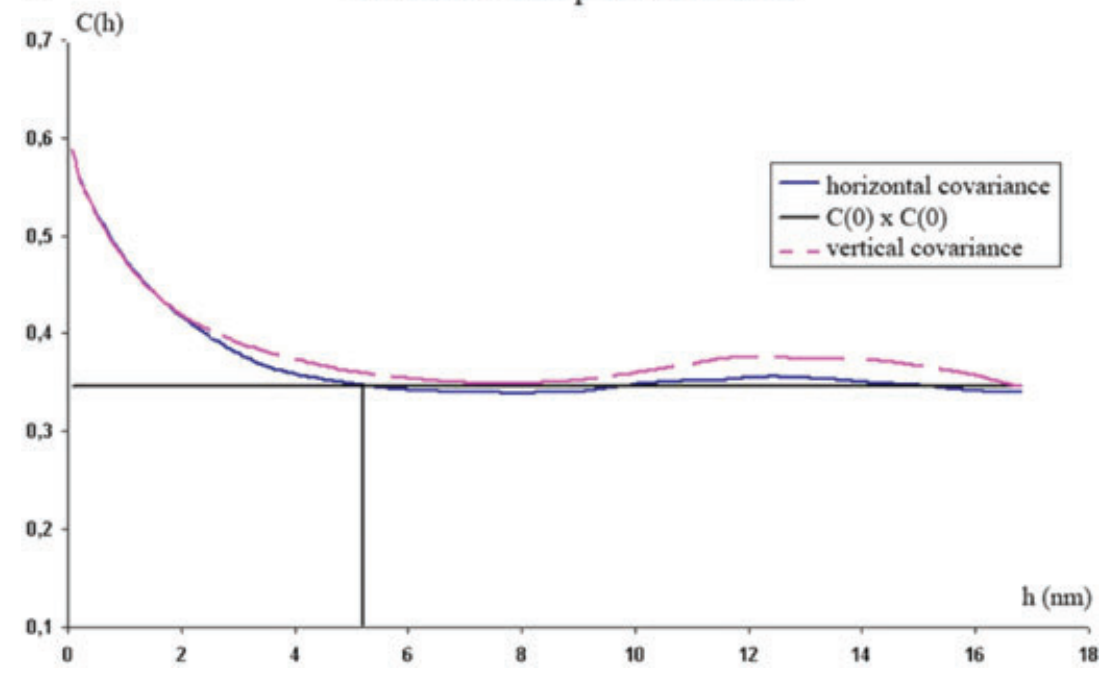

Covariance: nanoparticles of Ceria
)

Covariance: nanoparticles of Ceria
Fig. 8. Comparison of the average covariance functions measured horizontally and vertically on the images of sets 1, 2 and 3 (from top to bottom). The two curves (horizontal covariance and vertical covariance) are relatively close from each other in each case: the distribution of the ceria nanoparticles is isotropic. 


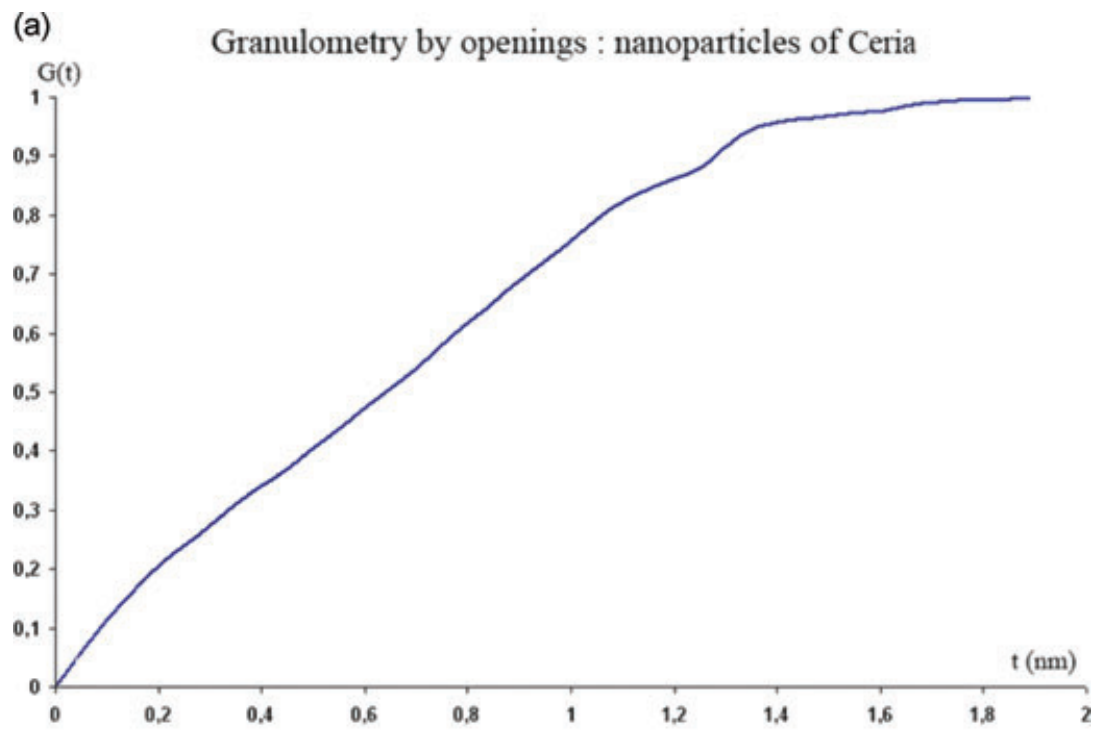

(b) Granulometry by openings : nanoparticles of Ceria

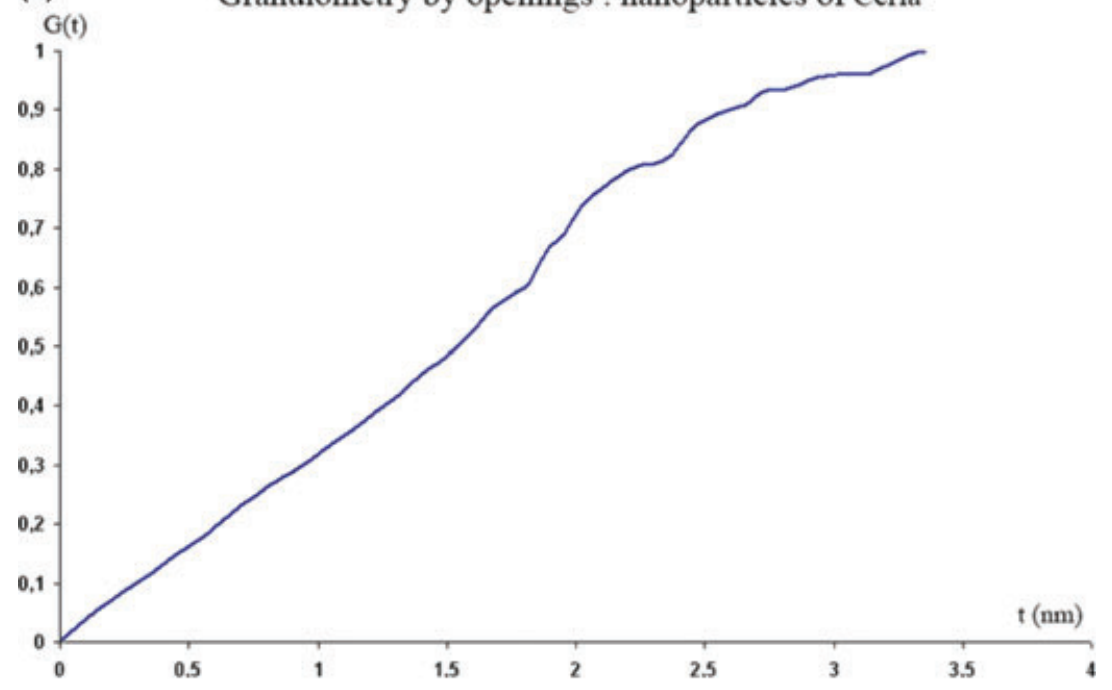

(c) Granulometry by openings : nanoparticles of Ceria

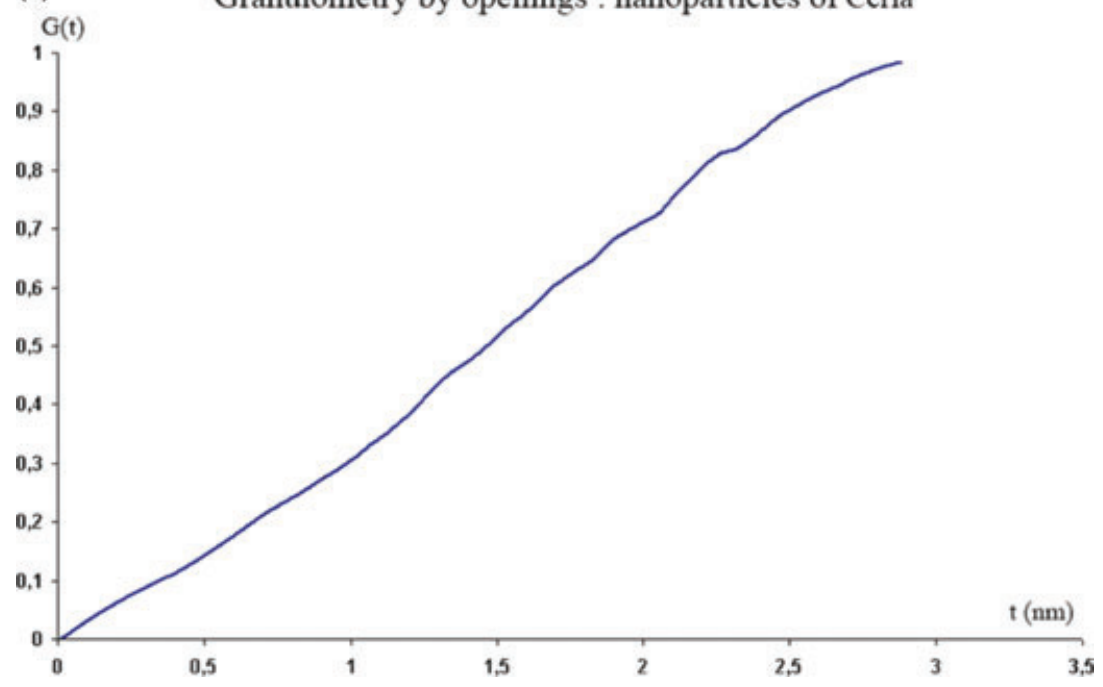

Fig. 9. Average size distribution functions $G(t)$ of the five images of sets 1, 2 and 3 (from top to bottom). 
(a)

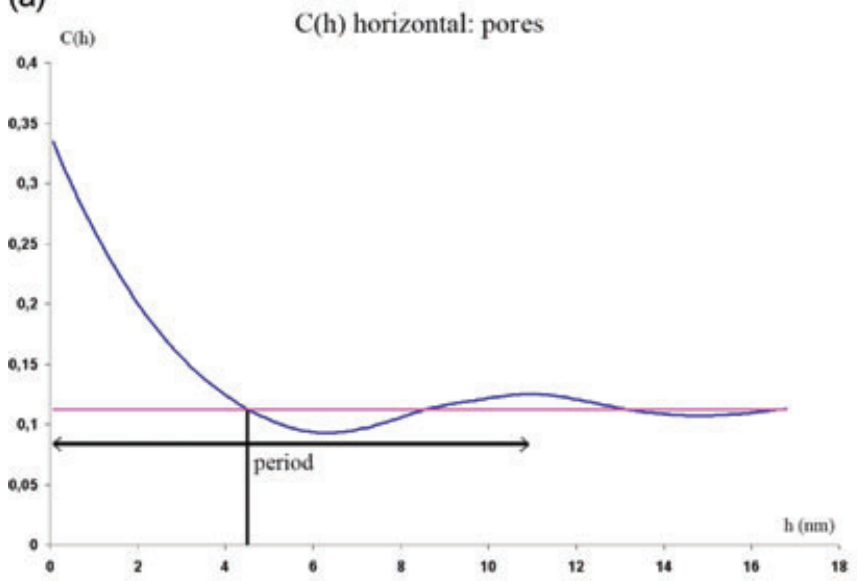

(c)

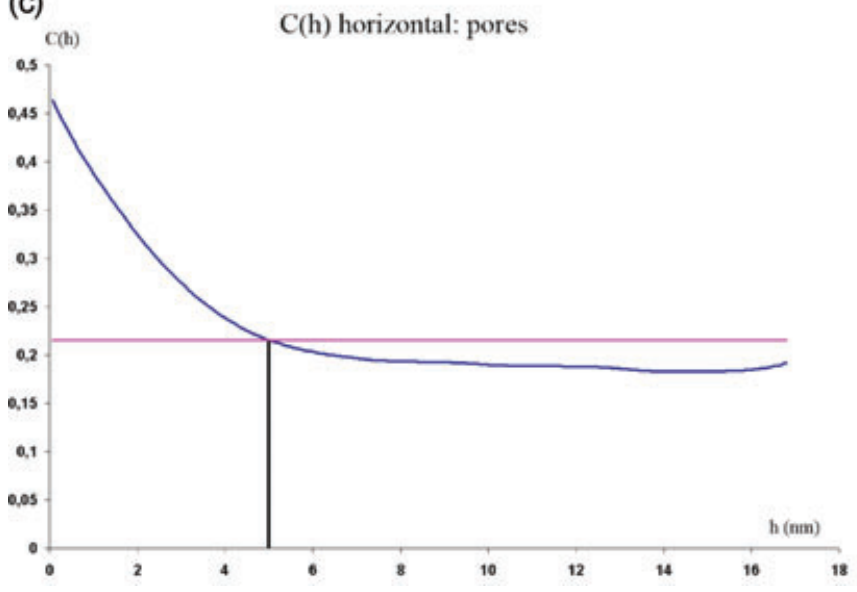

(b)

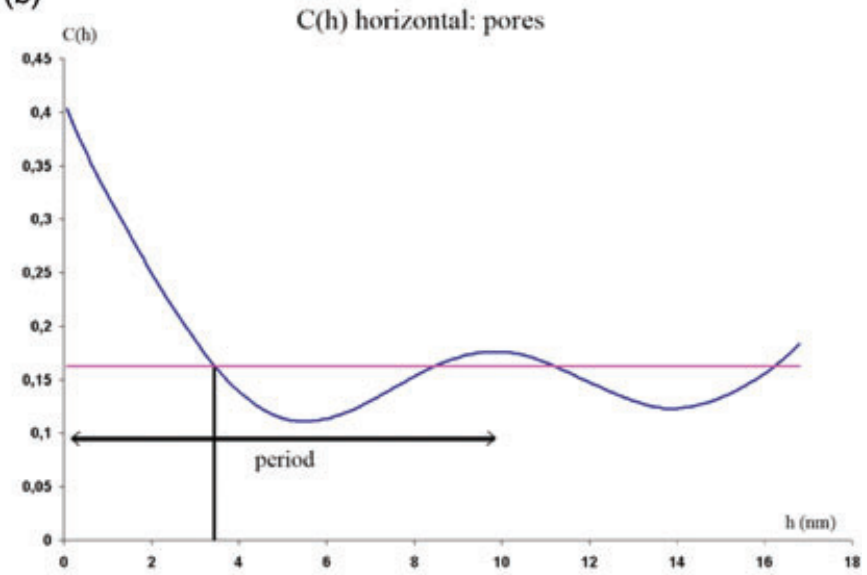

(d)

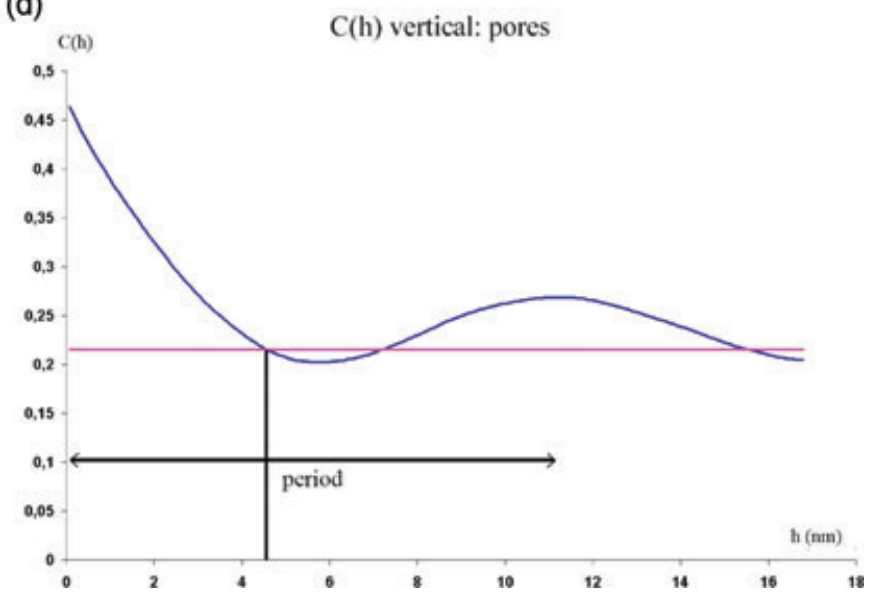

Fig. 10. (A) Horizontal covariance of the porous network of catalysts of sets 1 and 2 (from top to bottom). We can notice that the two catalysts present a quasi-periodic distribution of pores. (B) From top to bottom, curves of the horizontal and vertical covariance for set 3. We can notice a quasi-periodicity in one direction.

Table 1. Vv of pores $P$ and of ceria nanoparticles $C$ for the three sets of catalysts.

\begin{tabular}{llll}
\hline Catalysts & $\begin{array}{l}\text { Set 1 } \\
(\%)\end{array}$ & $\begin{array}{l}\text { Set 2 } \\
(\%)\end{array}$ & $\begin{array}{l}\text { Set 3 } \\
(\%)\end{array}$ \\
\hline Vv of pores $\mathrm{Vv}(P)$ & 33 & 40 & 46 \\
Vv of the ceria nanoparticles $\mathrm{Vv}(C)$ & 36 & 61 & 59 \\
Total $\mathrm{Vv}$ of ceria overlapping pores $\mathrm{Vv}(P \cap C)$ & 6 & 20 & 22 \\
Amount of ceria emerging in pores $\mathrm{Vv}(P \cap C) / \mathrm{Vv}(C)$ & 17 & 33 & 38 \\
$\mathrm{Vv}$ of pores containing ceria $\mathrm{Vv}(P \cap C) / \mathrm{Vv}(P)$ & 18 & 49 & 48
\end{tabular}

range of about $3.5-4.5 \mathrm{~nm}$, which is close to the result obtained by $\mathrm{N}_{2}$ adsorption $(7.5 \mathrm{~nm}$ ) (Chane-Ching et al., 2005). We also estimate that the mean size of the fields of ceria is about 4.4$5.3 \mathrm{~nm}$, which is identical to the result obtained by high-angle X-ray diffraction ( $5 \mathrm{~nm}$ ) (Chane-Ching et al., 2005).

An increase of the pore volume fraction is observed for composite materials displaying larger ceria nanoparticles volume fraction. Nevertheless, the pore diameter determined from TEM image analysis did not show any significant variation for various $\mathrm{CeO}_{2}$ concentrations in the composite. We suggest that the increase in pore volume could arise from the more rugged character of the surfaces observed at high $\mathrm{CeO}_{2}$ concentration as shown by the larger $\mathrm{CeO}_{2}$ volume fraction emerging in the pores and the larger total volume fraction of ceria overlapping pores for these composite materials. All these observations demonstrate that material synthetic routes involving insertion of nanoparticles is well suited for the preparation of high surface area composite materials displaying surface nanorugosity formed from protuberant $5 \mathrm{~nm} \quad \mathrm{CeO}_{2}$ nanoparticles.

\section{Conclusions}

Image analysis methods were presented to analyse automatically and quantitatively the morphology of three 


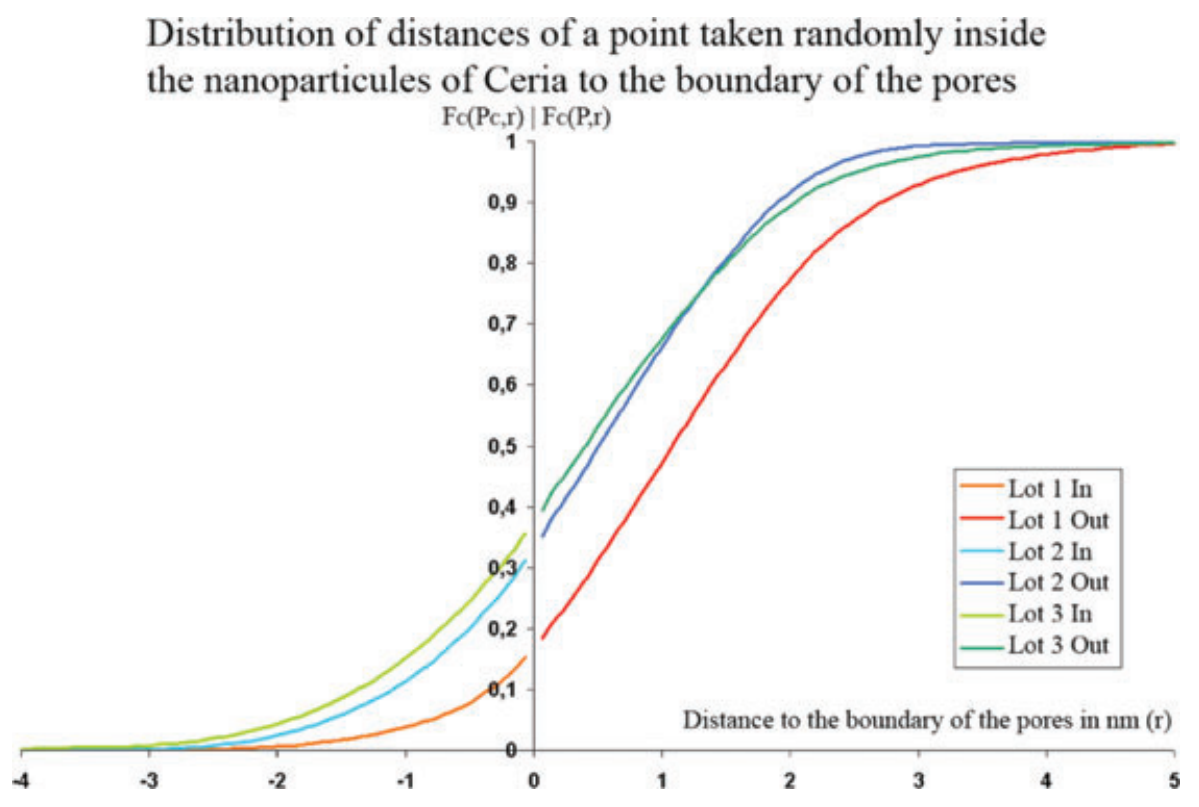

Fig. 11. $F_{\mathrm{C}}\left(P^{\mathrm{c}}, r\right)$ and $F_{\mathrm{C}}(P, r)$ for the three sets of catalysts.

high surface area silica-ceria catalysts imaged by TEM. These methods have allowed a segmentation of the components: pores, ceria nanoparticles and silica. The catalytic function is provided by the presence of ceria emerging into the pores. Distribution of the ceria nanoparticles, characterized by means of statistical and mathematical morphology tools and measurements, have highlighted the rugged character of the composite surfaces These image analysis methods can be used to characterize other materials observed under HRTEM conditions, in which the atomic organization presents oriented and/or periodic arrangement.

\section{References}

Chane-Ching, J.Y. (1999) Heat stable ordered mesostructured materials containing dispersed metal oxide nanocrystals. Patent number WO 2001, 032558.

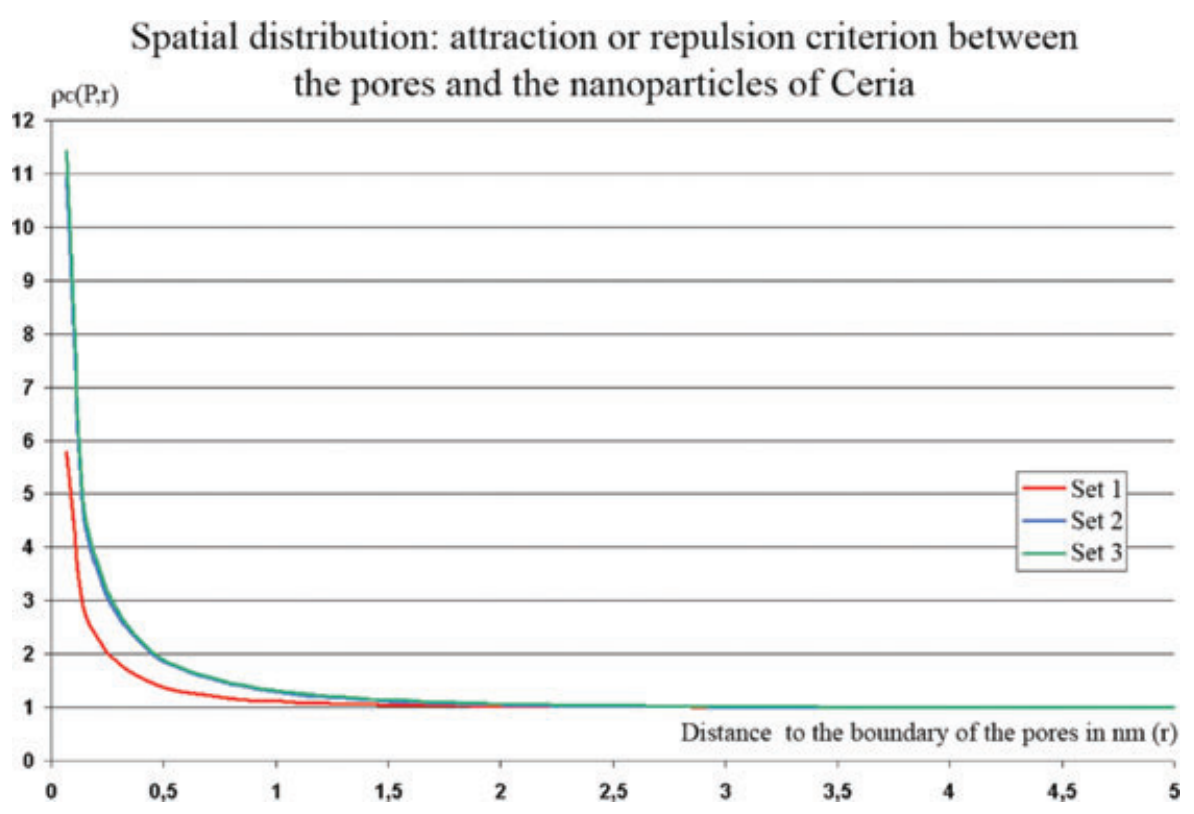

Fig. 12. $\rho_{\mathrm{C}}(P, r)$ for the three sets of catalysts. 
Table 2. Synthesis of the results of the analysis of the catalysts.

\begin{tabular}{|c|c|c|c|}
\hline $\begin{array}{l}\text { Analysis criterion } \\
\text { Pores network }\end{array}$ & $\begin{array}{l}\text { Set } 1 \\
\text { Quasi-periodic, } \\
\text { period } 11 \mathrm{~nm}\end{array}$ & $\begin{array}{l}\text { Set } 2 \\
\text { Quasi-periodic, } \\
\text { period } 10 \mathrm{~nm}\end{array}$ & $\begin{array}{l}\text { Set } 3 \\
\text { Quasi-periodic in one direction, } \\
\text { period } 11 \mathrm{~nm}\end{array}$ \\
\hline Vv of pores & $33 \%$ & $40 \%$ & $46 \%$ \\
\hline Mean size of pores & $4.5 \mathrm{~nm}$ & $3.5 \mathrm{~nm}$ & $4.5 \mathrm{~nm}$ \\
\hline Distribution of the ceria nanoparticles & Random - isotropic & Random - isotropic & Random - isotropic \\
\hline Vv of the ceria nanoparticles & $36 \%$ & $61 \%$ & $59 \%$ \\
\hline Size distribution of the fields of ceria & Quasi-uniform & Quasi-uniform & Quasi-uniform \\
\hline Mean size of the fields of ceria & $4.4 \mathrm{~nm}$ & $5.2 \mathrm{~nm}$ & $5.3 \mathrm{~nm}$ \\
\hline Total Vv of ceria overlapping pores & $6 \%$ & $20 \%$ & $22 \%$ \\
\hline Percentage of ceria emerging in pores & $17 \%$ & $33 \%$ & $38 \%$ \\
\hline $\mathrm{Vv}$ of pores containing ceria & $18 \%$ & $49 \%$ & $48 \%$ \\
\hline Distance to pore boundaries enclosing $50 \%$ of ceria & $1.1 \mathrm{~nm}$ & $0.5 \mathrm{~nm}$ & $0.4 \mathrm{~nm}$ \\
\hline
\end{tabular}

Chane-Ching, J.Y., Airiau, M., Balard, H. et al. (2005) Surface characterisation and properties of ordered arrays of $\mathrm{CeO}_{2}$ nanoparticles embedded in thin layers of $\mathrm{SiO}_{2}$ surfaces. Langmuir 21, 15681574.

Che, S., Garcia-Bennet, A.E., Kunieda, H., Sakamoto, K., Tatsumi, T., Terasaki, O.\& Yokoi, T. (2003) A novel Anionic surfactant templating route fro synthesizing mesoporous silica with unique structure. Nat. Mater. 2, 801-805.

Corma, A., Airiau, M., Chane-Ching, J.Y. \& Martinez, C. (2004a) Synthesis and catalytic properties of thermally and hydrothermally stable, high surface area $\mathrm{SiO}_{2}-\mathrm{CeO}_{2}$ mesostructured composite materials and their application for the removal of sulfur compounds from gasoline. $J$. Catalysis 224, 441-448.

Corma, A., Atienzar, P., Chane-Ching, J.Y. \& Garcia, H. (2004b) Hierarchically mesostructured doped $\mathrm{CeO}_{2}$ with potential for solar-cell use. Nat. Mater. 3, 394-397.

Jeulin, D. (1986) Study of spatial distributions in multicomponent structures by image analysis. Proc. 4th European Symposium of Stereology, Acta Stereologica 5/2, 233-239.

Jeulin, D. (1991) Morphological models of random structures and of change of scale (in French). Thèse de Doctorat d'Etat, Caen University, France.
Matheron, G. (1975) Random Sets and Integral Geometry. J. Wiley, New York.

Moreaud, M. (2006) Multi-scale morphological properties and prediction of the dielectric behaviour of nanocomposites (in French), pp. 23-41. Thesis, Paris School of Mines, France.

Nooney, R.I., Chen, Y., Josephs, R., Ostafin, A.E. \& Thirunavukkarasu, D. (2002) Synthesis of nanoscale mesoporous silica spheres with controlled particle size. Chem. Mater. 14, 4721-4728.

Otsu, N. (1979) A threshold selection method from gray level histograms. IEEE, Trans. Systems, Man and Cybernetics 9, 62-66.

Serra, J. (1982) Image Analysis and Mathematical Morphology. Academic Press, London.

Shinkai, S., Friggeri, A. \& Van Bommel, K.J.C. (2002) Organic templates for the generation of inorganic material. Angew. Chem. Ind. Ed. 42 , 980-999.

Thorel, A. \& Molins, R. (2003) Preliminary raport: Analysis of a silica-ceria catalyst with a new generation TEM (Tecnai 2OFST). Internal report, (in French). Center of Materials Sciences, MINES ParisTech.

Tian, B., Fan, J., Liu, X. et al. (2003) Self-adjusted synthesis or ordered stable mesoporous minerals by acid-base pairs. Nat. Mater. 2, 159163. 